\title{
Clinical Implications of SARS-CoV-2 Infection in the Viable Preterm Period
}

\author{
Moti Gulersen, MD, MSc ${ }^{1}$ Matthew J. Blitz, $\mathrm{MD}^{2}{ }^{2}$ \\ Weiwei Shan, PhD 3 Eran Bornstein, MD ${ }^{4}$

\footnotetext{
${ }^{1}$ Department of Obstetrics and Gynecology, North Shore University Hospital-Northwell Health, Manhasset, New York

2 Department of Obstetrics and Gynecology, Southside Hospital-

Northwell Health, Bay Shore, New York

3 Biostatistics Unit, Feinstein Institutes for Medical Research, Manhasset, New York

${ }^{4}$ Department of Obstetrics and Gynecology, Lenox Hill HospitalNorthwell Health, New York
}

Am J Perinatol 2020;37:1077-1083.
Burton Rochelson, MD ${ }^{1}$ Michael Nimaroff, MD ${ }^{1}$

Address for correspondence Moti Gulersen, MD, MSc, Department of Obstetrics and Gynecology, North Shore University Hospital, 300 Community Drive, Manhasset, NY 11030

(e-mail: mgulersen1@northwell.edu).

\section{Keywords \\ - SARS-CoV-2 \\ - COVID-19 \\ - coronavirus \\ - pregnancy \\ - preterm birth \\ - prematurity}

Objective This study aimed to determine the rate of preterm birth (PTB) during hospitalization among women diagnosed with severe acute respiratory syndrome coronavirus 2 (SARS-CoV-2) between 23 and 37 weeks of gestation and whether this rate differs by gestational age at diagnosis of infection.

Study Design Retrospective, cross-sectional study of all women diagnosed with SARS-CoV-2 infection between 23 and 37 weeks of gestation within a large integrated health system from March 13 to April 24, 2020. Cases with severe fetal structural malformations detected prior to infection were excluded. Women were stratified into two groups based on gestational age at diagnosis: early preterm ( $23^{0 / 7}$ to $33^{6 / 7}$ weeks) versus late preterm (34 to $36^{6 / 7}$ weeks). We compared the rate of PTB during hospitalization with infection between the two groups. Statistical analysis included use of Wilcoxon rank sum and Fisher exact tests, as well as a multivariable logistic regression. Statistical significance was defined as a $p$-value $<0.05$.

Results Of the 65 patients included, 36 (53.7\%) were diagnosed in the early preterm period and 29 (46.3\%) were diagnosed in the late preterm period. Baseline demographics were similar between groups. The rate of PTB during hospitalization with infection was significantly lower among women diagnosed in the early preterm period compared with late preterm (7/36 [19.4\%] vs. 18/29 [62\%], $p$-value $=0.001)$. Of the 25 patients who delivered during hospitalization with infection, the majority were indicated deliveries $(64 \%, 16 / 25)$. There were no deliveries $<33$ weeks of gestation for worsening coronavirus disease 2019 and severity of disease did not alter the likelihood of delivery during hospitalization with SARS-CoV-2 infection (adjusted odds ratio [aOR]: $0.64 ; 95 \%$ confidence interval $[\mathrm{Cl}]: 0.24-1.59$ ). Increased maternal age was associated with a lower likelihood of delivery during hospitalization with SARS-CoV-2 infection (aOR: 0.77; 95\% Cl: 0.58-0.96), while later gestational age at diagnosis of infection was associated with a higher likelihood of delivery during hospitalization (aOR: 2.9; 95\% Cl: 1.67-8.09). received

May 28, 2020 accepted after revision June 3, 2020 published online July 2, 2020
Copyright $@ 2020$ by Thieme Medical Publishers, Inc., 333 Seventh Avenue, New York, NY 10001, USA. Tel: +1(212) 760-0888.
DOI https://doi.org/

10.1055/s-0040-1713851. ISSN 0735-1631. 
Conclusion The likelihood of PTB during hospitalization with SARS-CoV-2 infection is significantly lower among women diagnosed in the early preterm period compared with late preterm. Most women with SARS-CoV-2 infection in the early preterm period recovered and were discharged home. The majority of PTB were indicated and not due to spontaneous preterm labor.

\section{Key Points}

- Preterm delivery is less likely among women diagnosed in the early preterm compared with late preterm.

- Most women infected in the early preterm period recovered and were discharged home undelivered.

- The majority of preterm birth were indicated and not due to spontaneous preterm labor.

On March 11, 2020, the World Health Organization declared the outbreak of the novel coronavirus, severe acute respiratory distress syndrome coronavirus 2 (SARS-CoV-2), a global pandemic. ${ }^{1}$ Since the first reported case, over 4 million people have been infected with nearly 300,000 dying from complications related to the novel coronavirus disease (COVID-19) worldwide. $^{2}$ While clinical sequelae after infection varies, ranging from asymptomatic carriers to critical illness and death, the majority of infected individuals recover. ${ }^{3}$ Nevertheless, reports outlining the disease course in pregnant women, particularly in the preterm period, are limited, leaving health care providers with a continued challenge of determining optimal management strategies.

Preterm birth (PTB) remains the leading cause of neonatal morbidity and mortality in the United States. ${ }^{4-8}$ Preterm delivery has been reported among infants born to women with SARS-CoV-2 infection during pregnancy. ${ }^{9-12}$ Furthermore, many of the reported early deliveries appear to have been indicated and not due to spontaneous preterm labor. ${ }^{11}$ An understanding of the clinical implications of SARS-CoV-2 infection in the viable preterm period and risk of preterm birth is of importance, as management decisions are carefully made based on weighing risks of maternal disease with risk of prematurity-related complications. Therefore, the objectives of this study were to determine the rate of PTB during infection among women diagnosed with SARS-CoV-2 in a hospital setting between 23 and 37 weeks of gestation, and whether this rate differed by gestational age at diagnosis.

\section{Materials and Methods}

This was a retrospective, cross-sectional study of all women diagnosed with SARS-CoV-2 infection between 23 and 37 weeks of gestation. The study was conducted at seven hospitals within the Northwell Health system in New York: North Shore University Hospital (Manhasset, NY), Long Island Jewish Medical Center (Queens, NY), Long Island Jewish Forest Hills (Forest Hills, NY), Huntington Hospital (Huntington, NY), Southside Hospital (Bay Shore, NY), Lenox Hill Hospital (New York, NY), and Staten Island University Hospital (Staten Island, NY). The Northwell Health Institutional Review Board approved this study as minimal-risk research using data collected for routine clinical practice and waived the requirement for informed consent. A subset of patients in this study were included in previous studies characterizing hospitalizations with SARS-CoV-2 infection within the Northwell Health system. ${ }^{13,14}$

Women who had laboratory-confirmed SARS-CoV-2 infection after hospital presentation from March 13 to April 24, 2020 were eligible for inclusion. This included women who were evaluated in the emergency department or labor and delivery unit, and were either admitted or discharged home based on their clinical condition. Diagnosis of infection was confirmed by use of qualitative real-time polymerase chain reaction (PCR) on maternal nasopharyngeal swab specimens. Beginning on April 2, universal testing for SARS-CoV-2 infection was implemented for all obstetrical patients admitted to Labor and Delivery and antepartum units in all participating sites. Prior to April 2, testing was performed based on the clinical suspicion for SARS-CoV-2 infection (i.e., fever, flu-like symptoms, travel history, known, or suspected exposure). Patients with severe fetal structural anomalies diagnosed before or at presentation were excluded. A detailed review of each medical record was performed. Maternal characteristics such as age, weight, ethnicity, parity, and comorbidities-if present-were collected. Comorbid conditions included asthma, chronic hypertension, type 2 diabetes mellitus, HIV, and autoimmune disorders. Additionally, subjective and objective data relating to disease illness, if available, were obtained including presence or absence of symptoms (i.e., fever/chills, cough, dyspnea, chest pain, myalgias, nausea and/or vomiting, diarrhea, and headache), documented temperature $\geq 100.4^{\circ} \mathrm{F}$ and treatment regimen.

Disease severity was classified as either: symptoms not directly related to SARS-CoV-2 infection; mild, severe or critical SARS-CoV-2-related symptoms based on the defined criteria from the Chinese Center for Disease Control and Prevention. ${ }^{15}$ Mild cases were defined as those without pneumonia or with mild pneumonia. Severe cases were defined as those with dyspnea, respiratory rate $\geq 30$ breaths per minute, blood oxygen saturation $\leq 93 \%$ on room air, partial pressure of arterial oxygen to fraction of inspired oxygen $<300$, and/or lung infiltrates $>50 \%$ within 24 to 48 hours of symptom onset. Critical cases were defined as 
those that exhibited respiratory failure, septic shock, and/or multiple organ dysfunction or failure. Obstetrical symptoms not necessarily related to SARS-CoV-2 infection were reviewed, including contractions, leakage of amniotic fluid, or vaginal bleeding. Patient disposition was determined by the caring provider and characterized as outpatient (not admitted), inpatient antepartum, inpatient intensive care unit (ICU), or inpatient labor and delivery.

Cases were stratified into two groups based on gestational age at diagnosis of SARS-CoV-2 infection: early preterm (23 to $33^{6 / 7}$ weeks of gestation) versus late preterm (34 to $36^{6 / 7}$ weeks of gestation). All patients had an estimation of gestational age and due date established or confirmed by a firsttrimester ultrasound. The primary outcome was PTB during hospitalization with SARS-CoV-2 infection. Patients in each group who did not deliver during hospitalization with SARSCoV-2 infection were further characterized as either delivered at $<37$ weeks, delivered at $\geq 37$ weeks, ongoing pregnancy at $<37$ weeks, or ongoing pregnancy at $\geq 37$ weeks. The overall rate of PTB could not be determined as there were ongoing pregnancies, representing the most favorable clinical scenario in terms of risk for PTB, in patients who were discharged home after infection and still pregnant at the time of this review.

Statistical analysis included use of Wilcoxon rank sum test and Fisher's exact test for comparison of categorical and continuous variables. To determine if the observed difference in PTB during hospitalization with SARS-CoV-2 infection was attributable to differences in baseline characteristics, a multivariable logistic regression was performed. This included multiple regression models with combinations of predictors such as maternal age, nulliparity, body mass index (BMI), race/ethnicity, presence of comorbid conditions, gestational age at diagnosis of SARS-CoV-2 infection, and severity of disease. Severity of disease was constructed using a linear scale from 0 to 3, where 0 was symptoms not directly related to SARS-CoV-2 infection, and 3 was critical symptoms related to SARS-CoV-2 infection. The best model was selected based on the fitness determined by Akaike information criterion and log likelihood. Odds ratios (OR) were calculated with $95 \%$ confidence intervals (CI). Statistical significance was defined as a $p$-value $<0.05$.

\section{Results}

Our population included 67 patients with SARS-CoV-2 infection between 23 and 37 weeks of gestation. Two cases with multiple fetal structural malformations that were detected prior to SARS-CoV-2 infection were excluded from the analysis. The first case was a 30 -year-old gravida 2 para 0010 who was diagnosed with severe COVID-19 at 31 weeks and 1 day of gestation. Multiple fetal anomalies were detected on her anatomical survey and fetal echocardiogram. These included bilateral fetal neck masses, muscular ventricular septal defect, absent ductus venosus, hypoplastic aortic arch, bilateral fetal pyelectasis, and polyhydramnios. Cell-free DNA reported as low risk, and the patient declined amniocentesis with microarray analysis despite the suspicion of fetal syndrome. She was diagnosed with an intrauterine fetal demise at 36 weeks of gestation. The patient declined autopsy and cytogenetic evaluation has not resulted. The second case was a 37-year-old gravida 3 para 2002 who was diagnosed with mild COVID-19 at 35 weeks and 4 days of gestation after presenting with preterm prelabor rupture of membranes (PPROM) and an intrauterine fetal demise. Her prenatal course was significant for abnormal cell-free DNA screening, high-risk for trisomy 13. Abnormal findings were found on ultrasound: severe, early-onset fetal growth restriction, hypoplastic cerebellum, absent cavum septum pellucidum, echogenic kidneys, intrahepatic calcifications, and cardiomegaly. The patient declined invasive prenatal diagnostic testing.

After applying our exclusion criteria, 65 patients comprised the study cohort and were further analyzed. Of the 65 patients, 36 (53.7\%) were diagnosed in the early preterm period (23 to 34 weeks of gestation) and 29 (46.3\%) were diagnosed in the late preterm period ( 34 to 37 weeks of gestation). Maternal age, parity, BMI, race/ethnicity, and the presence of comorbid conditions were similar between both groups (-Table 1 ). Subjective fever/chills and cough were the most common symptoms reported in both groups and were more frequently reported among patients diagnosed in the early preterm period compared with late preterm (fever/chills: 77.8 vs. $51.7 \%, p$-value $=0.04$; cough: 83.3 vs. $51.7 \%, p$-value $=0.008$; -Table 1). Severity of disease was significantly different between both groups ( $p=0.03$; - Table 2 ). For patients diagnosed in the early preterm period, $8.3 \%(3 / 36)$ were without symptoms related to SARS-CoV-2 infection, 33.3\% (12/36) had mild disease, $44.5 \%$ (16/36) had severe disease, and 13.9\% (5/ 36) had critical disease. For patients diagnosed in the late preterm period, $37.9 \%(11 / 29)$ were without symptoms related to SARS-CoV-2 infection, $27.6 \%$ (8/29) had mild disease, $27.6 \%$ (8/29) had severe disease, and 6.9\% (2/29) had critical disease. Patient disposition was also significantly different between both groups ( $p=0.02 ;$ - Table 2 ). For patients diagnosed in the early preterm period, $36.1 \%$ (13/36) were admitted to the inpatient antepartum service, $11.1 \%(4 / 36)$ were admitted to the ICU, $16.7 \%$ (6/36) were admitted to labor and delivery and $36.1 \%(13 / 36)$ were not admitted. For patients diagnosed in the late preterm period, $17.3 \%$ (5/29) were admitted to the inpatient antepartum service, $3.4 \%$ (1/29) were admitted to the ICU, $51.7 \%$ (15/29) were admitted to labor and delivery, and $27.6 \%$ (8/29) were not admitted. There was no significant difference in pharmacologic treatment of disease between both groups (-Table 2).

The rate of PTB during hospitalization with SARS-CoV-2 infection was significantly lower among women diagnosed in the early preterm period compared with late preterm (7/ 36 [19.4\%] vs. 18/29 [62\%], $p$-value $=0.001$; - Table 2 ). Of the seven patients who delivered after diagnosis of infection in the early preterm period, four symptomatic patients with COVID-19 were delivered due to worsening disease between 33 and 34 weeks of gestation. The remaining three were delivered after the diagnosis of preeclampsia with severe features, spontaneous preterm labor, and chorioamnionitis in the setting of prolonged PPROM. Of the 18 patients who delivered after diagnosis of infection in the later preterm period, five symptomatic patients were delivered due to 


\begin{tabular}{|c|c|c|c|}
\hline & $23-34$ weeks $(n=36)$ & $34-37$ weeks $(n=29)$ & $p$-Value \\
\hline Maternal age, median (IQR) (y) & $31.5(29-34)$ & $32(30-35)$ & 0.65 \\
\hline BMI, median (IQR) (kg/m²) & $32(27.2-36.6)$ & $31(28-36.3)$ & 1 \\
\hline \multicolumn{4}{|l|}{ Race or ethnic group, $n$ (\%) } \\
\hline Non-Hispanic White & $7(19.4)$ & $8(27.6)$ & \multirow[t]{5}{*}{0.39} \\
\hline Non-Hispanic Black & $6(16.7)$ & $8(27.6)$ & \\
\hline Hispanic & $17(47.2)$ & $7(24.1)$ & \\
\hline Asian & $4(11.1)$ & $3(10.3)$ & \\
\hline Other/unknown & $2(5.6)$ & $3(10.3)$ & \\
\hline Nulliparous, $n(\%)$ & $12(33.3)$ & $11(37.9)$ & 0.8 \\
\hline GA at diagnosis, median (IQR) (wk) & $30.1(27-33)$ & $35.3(34.7-36.1)$ & $<0.001$ \\
\hline Presence of comorbidity ${ }^{\mathrm{a}}, n(\%)$ & $9(25)$ & $2(6.9)$ & 0.09 \\
\hline \multicolumn{4}{|l|}{ SARS-CoV-2 related symptoms, $n(\%)$} \\
\hline Fever/chills & $28(77.8)$ & $15(51.7)$ & 0.04 \\
\hline Cough & $30(83.3)$ & $15(51.7)$ & 0.008 \\
\hline Dyspnea & $18(50)$ & $9(31)$ & 0.138 \\
\hline Chest pain & $2(5.6)$ & $2(6.9)$ & 1 \\
\hline Myalgias & $14(38.9)$ & $6(20.7)$ & 0.18 \\
\hline Nausea/vomiting & $6(16.7)$ & $2(6.9)$ & 0.28 \\
\hline Diarrhea & $2(5.6)$ & $0(0)$ & 0.5 \\
\hline Headache & $4(11.1)$ & $2(6.9)$ & 0.68 \\
\hline Symptoms not directly related to SARS-CoV-2 infection, $n$ (\%) & $3(8.3)$ & $11(37.9)$ & 0.006 \\
\hline Temperature $\geq 100.4^{\circ} \mathrm{F}$ on evaluation, $n(\%)$ & $20(61.1)$ & $21(72.4)$ & 0.43 \\
\hline
\end{tabular}

Abbreviations: BMI, body mass index; GA, gestational age; IQR, interquartile range; SARS-CoV-2, severe acute respiratory syndrome coronavirus 2. ${ }^{a}$ Comorbid conditions included asthma, chronic hypertension, type 2 diabetes mellitus, HIV, and autoimmune disorders.

\begin{tabular}{|c|c|c|c|}
\hline & $23-34$ weeks $(n=36)$ & $34-37$ weeks $(n=29)$ & $p$-Value \\
\hline Delivery during admission, $n(\%)$ & $7(19.4)$ & $18(62)$ & 0.001 \\
\hline Delivered to date, $n(\%)$ & $20(55.6)$ & $27(93.1)$ & 0.002 \\
\hline \multicolumn{4}{|l|}{ SARS-CoV-2 severity, $n$ (\%) } \\
\hline Positive test with no typical SARS-CoV-2 symptoms & $3(8.3)$ & $11(37.9)$ & \multirow[t]{4}{*}{0.03} \\
\hline Mild & $12(33.3)$ & $8(27.6)$ & \\
\hline Severe & $16(44.5)$ & $8(27.6)$ & \\
\hline Critical & $5(13.9)$ & $2(6.9)$ & \\
\hline \multicolumn{4}{|l|}{ Disposition, $n(\%)$} \\
\hline Outpatient: not admitted & $13(36.1)$ & $8(27.6)$ & \multirow[t]{4}{*}{0.02} \\
\hline Inpatient: antepartum & $13(36.1)$ & $5(17.3)$ & \\
\hline Inpatient: L\&D & $6(16.7)$ & $15(51.7)$ & \\
\hline Inpatient: ICU & $4(11.1)$ & $1(3.4)$ & \\
\hline Length of hospital stay, median (IQR) (d) & $4(2-7)$ & $4(3-4)$ & 0.8 \\
\hline \multicolumn{4}{|l|}{ Treatment, $n(\%)$} \\
\hline Antibiotics & $9(25)$ & $5(17.3)$ & 0.55 \\
\hline Antimalarial & $10(27.8)$ & $7(24.1)$ & 0.78 \\
\hline Steroids & $4(11.1)$ & $0(0)$ & 0.12 \\
\hline Anti-interleukin & $4(11.1)$ & $0(0)$ & 0.12 \\
\hline None & $12(33.3)$ & $22(75.9)$ & 0.001 \\
\hline
\end{tabular}

Abbreviations: ICU, intensive care unit; IQR, interquartile range; L\&D, labor and delivery; SARS-CoV-2, severe acute respiratory syndrome coronavirus 2. 


\begin{tabular}{llll|}
\hline $\begin{array}{l}\text { Table } 3 \\
\text { associated with likelihood of preterm birth during } \\
\text { hospitalization with SARS-CoV-2 infection }\end{array}$ & \\
\hline Variable & $\begin{array}{l}\text { Adjusted } \\
\text { odds ratio }\end{array}$ & $\begin{array}{l}95 \% \\
\text { confidence } \\
\text { interval }\end{array}$ & $p$-Value \\
& 0.77 & $0.58-0.96$ & 0.04 \\
Maternal age & 1.07 & $0.92-1.28$ & 0.41 \\
$\begin{array}{l}\text { Body mass index } \\
\text { Nulliparity }\end{array}$ & 0.5 & $0.04-4.1$ & 0.55 \\
$\begin{array}{l}\text { Presence of } \\
\text { comorbid conditions }\end{array}$ & 1.19 & $0.11-14.12$ & 0.89 \\
$\begin{array}{l}\text { Race/ethnicity: } \\
\text { Hispanic }\end{array}$ & 1.92 & $0.06-46.5$ & 0.69 \\
$\begin{array}{l}\text { Race/ethnicity: } \\
\text { Non-Hispanic White }\end{array}$ & 0.26 & $0.01-5.5$ & 0.42 \\
$\begin{array}{l}\text { Race/ethnicity: } \\
\text { Non-Hispanic Black }\end{array}$ & 0.07 & $0-1.34$ & 0.12 \\
$\begin{array}{l}\text { Disease severity } \\
\begin{array}{l}\text { Gestational age at } \\
\text { diagnosis of infection }\end{array}\end{array}$ & 0.64 & $0.24-1.59$ & 0.34 \\
\hline
\end{tabular}

Abbreviation: SARS-CoV-2, severe acute respiratory syndrome coronavirus 2.

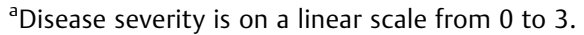

Notes: Reference group for race/ethnicity is Asian.

Maternal age, body mass index, and gestational age at diagnosis of infection were entered into the model as continuous variables.

worsening disease. The remaining 13 were delivered due to fetal growth restriction (2), spontaneous preterm labor (4), PPROM (3), preeclampsia with severe features (2), placenta previa, (1) and placenta accreta (1). Therefore, of the 25 patients who delivered during the course of their infection, the majority $(64 \%, 16 / 25)$ were indicated deliveries.

On multivariable analysis, increased maternal age was associated with a lower likelihood of delivery during hospitalization with SARS-CoV-2 infection (adjusted odds ratio [aOR]: 0.77; 95\% confidence interval [CI]: 0.58-0.96), while later gestational age at diagnosis of infection was associated with a higher likelihood of delivery during hospitalization (aOR: 2.9; 95\% CI: 1.67-8.09; - Table 3). BMI, race/ethnicity, presence of comorbid conditions, or severity of disease was not associated with likelihood of PTB during the course of infection (- Table 3).

To date, $47(72.3 \%, 47 / 65)$ patients have delivered: 20 $(55.6 \%, 20 / 36)$ after diagnosis in the early preterm period and $27(93.1 \%, 27 / 29)$ after diagnosis in the later preterm period (-Table 2). Thus, 18 pregnancies, including many (44.4\%) of the ones diagnosed with infection in the early preterm period, are ongoing. Of the 29 patients who did not deliver during hospitalization with SARS-CoV-2 infection after diagnosis in the early preterm period, $5(17.3 \%, 5 / 29)$ delivered at $<37$ weeks and $8(27.6 \%, 8 / 29)$ delivered at $\geq 37$ weeks. Seven patients $(24.1 \%, 7 / 29)$ have ongoing pregnancies at $<37$ weeks and $9(31 \%, 9 / 29)$ have ongoing pregnancies at $\geq 37$ weeks. Of the nine patients who did not deliver during hospitalization with SARS-CoV-2 infection after diagnosis in the late preterm period, none delivered at $<37$ weeks and 7
(77.8\%, 7/9) delivered at $\geq 37$ weeks. Two patients (22.2\%, 2 / 9) have ongoing pregnancies at $\geq 37$ weeks.

\section{Discussion}

The results of our study illustrate several important findings. First, the likelihood of preterm delivery during hospitalization with SARS-CoV-2 infection is significantly lower among women diagnosed in the early preterm period compared to those diagnosed in the late preterm period. Second, the majority of the women infected in the early preterm period recovered from SARS-CoV-2 infection and were discharged home undelivered. Third, of the deliveries that occurred in the preterm period during infection, the majority were indicated and not due to spontaneous preterm labor.

The clinical course of SARS-CoV-2 infection in pregnant women, particularly in the viable preterm period, is not well known. Of the nine symptomatic patients reported in the first case series by Chen et al in March 2020, four were diagnosed and delivered in the preterm period (all after 36 weeks of gestation):two were medically indicated due to hypertensive disorders of pregnancy and two were spontaneous after presenting in preterm labor or PPROM. ${ }^{16}$ Since then cohort studies reporting outcomes of infected preterm patients, both symptomatic and those without symptoms related to SARS-CoV-2 infection, have been limited. Lokken et al recently reported obstetrical outcomes in a U.S. cohort of 46 pregnant women with SARS-CoV-2 from Washington State. ${ }^{10}$ The majority of their cohort was symptomatic (93.5\%) and 33 of the 46 cases were diagnosed between the 20 and 37 weeks of gestation. ${ }^{10}$ Of those 33 preterm patients, only one delivered during the course of infection at 33 weeks of gestation due to worsening respiratory status. ${ }^{10}$ Using the same established clinical criteria for severity of disease, $15.2 \%$ of their cohort had severe and/or critical COVID-19. ${ }^{10}$ The higher rate of severe and critical disease, as well as those without symptoms typically related to SARS$\mathrm{CoV}-2$ infection that tested positive after presenting for obstetrical indications, may have contributed to the much higher rate of PTB during course of infection seen in our study. In a multicenter cohort study of 64 hospitalized pregnant women with severe and critical COVID-19 in the United States, the rate of preterm delivery $<37$ weeks was $29.7 \%{ }^{11}$ Of the 31 pregnant women with severe and critical COVID-19 in our study, the rate of preterm delivery during hospitalization with infection was similar at $29 \%$. These rates may be underestimated, as a large number of patients in both studies had ongoing pregnancies at the time of reporting.

In our adjusted analysis, the likelihood of PTB during hospitalization with SARS-CoV-2 infection depended on maternal age and gestational age. The higher likelihood of preterm delivery associated with increasing gestational age was expected, as PTB becomes more likely as gestational age advances. ${ }^{17}$ However, as we did not have a control group, it is unclear whether the risk is higher or lower in the setting of SARS-CoV-2 infection. The lower likelihood of preterm delivery associated with increasing maternal age was unexpected. Older age in the nonpregnant population has been 
demonstrated as a risk factor for adverse outcomes associated with SARS-CoV-2 infection. ${ }^{18}$ Nevertheless, age as a risk factor for adverse outcomes in the pregnant population requires further study.

We stratified our cohort into two different gestational age groups, early preterm and late preterm period, for several reasons. Preterm women with COVID-19 pose a clinical dilemma for providers, as interventions are carefully decided and based on weighing maternal benefit of delivery with fetal risks of prematurity. It remains unclear if delivery in the setting of severe or critical maternal illness with COVID-19 confers maternal benefit. However, it is known that prematurityrelated complications correlate with gestational age and such complications are more frequently observed in the early preterm period compared with late preterm period. ${ }^{19}$ All nine women in our cohort who delivered due to worsening maternal respiratory status in the setting of COVID-19 were delivered between 33 weeks and 36 weeks and 6 days of gestation, and were discharged home after recovery. Of these nine neonates, one had respiratory distress syndrome and cultureproven sepsis. The remaining eight were discharged home without complications. Obstetric outcomes in the early preterm period and late preterm period were recently reported in a study by Yan et al in China. ${ }^{12}$ In their study cohort that included 116 pregnant women with COVID-19 pneumonia (laboratory-confirmed or clinically diagnosed), 10 cases presented between 24 and 34 weeks of gestation and 22 cases presented between 34 and 37 weeks of gestation. ${ }^{12}$ of the 10 cases in their early preterm period, 2 (20\%) had PTB during their course of infection: 1 at 28 weeks due to severe pneumonia and another at 31 due to multiple gestation. ${ }^{12}$ These results are similar to ours and provide reassuring data that the majority of women diagnosed in the early preterm period recover from infection and are discharged home undelivered. Furthermore, our data illustrate that there were no deliveries prior to 33 weeks of gestation for worsening COVID-19 and that severity of disease did not alter the likelihood of delivery during hospitalization with SARS-CoV-2 infection. Those delivered at 33 weeks of gestation for worsening disease likely reflect the proximity to 34 weeks, which were the gestational age at which delivery of patients with SARS-CoV-2-related symptoms were considered at our institution.

The most common reported SARS-CoV-2 related symptoms among patients in our study included fever/chills and cough. Early preterm diagnosis was associated with a higher rate of these symptoms. This was likely reflective of the fact that most patients presenting in the later preterm period had obstetrical symptoms not necessarily related to SARS-CoV-2 infection and were diagnosed based on universal testing. Nonetheless, our data are not sufficient to determine whether a different symptom pattern exists at a different gestational age of infection.

There are several strengths to our study. To our knowledge and based on a review of the literature, this is the first study investigating clinical outcomes exclusively among women with laboratory confirmed SARS-CoV-2 infection in the viable preterm period. Additionally, our multicenter cohort is derived from New York, where the total number of novel coronavirus infections is highest worldwide, and thus, the largest study of preterm patients reported to date. Lastly, all providers were from the same health system, where management strategies regarding delivery of antepartum patients with COVID-19 were standardized by Maternal-Fetal Medicine specialists and clinical practice was relatively homogeneous. Our population, which is derived from a health system that cares for women with over 30,000 deliveries annually, is diversified in terms of maternal race, ethnicity, demographics, and includes patients from a wide geographical area in New York State.

There are some limitations to this study. Given the paucity of data regarding COVID-19 in pregnancy and urgent need for more data, we report our experience while several of the pregnancies are ongoing and remain undelivered. Thus, the actual PTB rate is unknown. It is also possible that patients may have delivered at an institution outside of our health system. Furthermore, this cohort only included women who presented to a hospital, whether symptomatic or without symptoms related to SARS-CoV-2 infection, and as a result, likely underestimates the true prevalence of infection in viable preterm patients. With the introduction of systemwide universal testing on labor and delivery, the majority of PTBs that occurred during infection were in those without typical symptoms related to SARS-CoV-2 infection that had presented with obstetrical complaints $(13 / 25,52 \%)$, likely contributing to the high rate of prematurity within our cohort. While we reported various pharmacological therapies given to infected patients, recommendations regarding which treatment modality is optimal or when it is indicated cannot be made based on our data.

In conclusion, our data add to the limited existing literature regarding clinical outcomes of SARS-CoV-2 infection in the viable preterm period. Our data are reassuring, suggesting that the risk of preterm delivery during infection with SARS-CoV-2 is significantly lower among women diagnosed in the early preterm period compared with the late preterm period and that the majority of these women recovered from infection and were discharged home. Furthermore, of the deliveries that occurred in the preterm period during infection, the majority were indicated and not due to spontaneous preterm labor. This represents an important finding when counseling women regarding the risks associated with SARS$\mathrm{CoV}-2$ infection in the viable preterm period. It is not clear whether the obstetrical indications for preterm delivery observed in our cohort, such as preeclampsia and PPROM, may have been related to the SARS-CoV-2 infection or an independent complication in these women. Experts around the world are continuing to refine their management strategies based on available data. Future research from large cohorts is needed to better understand the maternal-fetal dilemma in this select patient population.

Conflict of Interest

None declared.

Acknowledgments

The authors would like to acknowledge the contributions of the Northwell Health COVID-19 Research Consortium. 


\section{References}

1 World Health Organization. WHO coronavirus disease (COVID-19) situation report - 51. Available at: https://www.who.int/docs/ default-source/coronaviruse/situation-reports/20200311-sitrep51-covid-19.pdf?sfvrsn=1ba62e57_10. Accessed May 14, 2020

2 World Health Organization. WHO coronavirus disease (COVID-19) situation report - 115. Available at: https://www.who.int/docs/ default-source/coronaviruse/situation-reports/20200514-covid-19 -sitrep-115.pdf?sfvrsn=3fce8d3c_6. Accessed May 14, 2020

3 World Health Organization. WHO coronavirus disease 2019 (COVID-19) situation report - 46. Available at: https://www. who.int/docs/default-source/coronaviruse/situation-reports/ 20200306-sitrep-46-covid-19.pdf?sfvrsn=96b04adf_4. Accessed May 14, 2020

4 United Nations Children's Fund (UNICEF). Committing to child survival: a promise renewed. Progress Report 2014. New York, NY: UNICEF; 2014

5 United Nations Inter-agency Group for Child Mortality Estimation (UN IGME). Levels \& trends in child mortality. Report 2014. New York, NY: UNICEF; 2014

6 Savitz DA, Blackmore CA, Thorp JM. Epidemiologic characteristics of preterm delivery: etiologic heterogeneity. Am J Obstet Gynecol 1991;164(02):467-471

7 Martin JA, Hamilton BE, Sutton PD, Ventura SJ, Mathews TJ, Osterman MJ. Births: final data for 2008. Natl Vital Stat Rep 2010;59(01):1-72, 3-71

8 Hamilton BE, Hoyert DL, Martin JA, Strobino DM, Guyer B. Annual summary of vital statistics: 2010-2011. Pediatrics 2013;131(03): 548-558

9 Breslin N, Baptiste C, Gyamfi-Bannerman C, et al. Coronarvirus disease 2019 infection among asymptomatic and symptomatic pregnant women: two weeks of confirmed presentations to an affiliated pair of New York City hospitals. Am J Obstet Gynecol MFM 2020. Doi: 10.1016/j.ajogmf.2020.100118
10 Lokken EM, Walker CL, Delaney S, et al. Clinical characteristics of 46 pregnant women with a SARS-CoV-2 infection in Washington state. Am J Obstet Gynecol 2020;S0002-9378(20)30558-5;

11 Pierce-Williams RAM, Burd J, Felder L, et al. Clinical course of severe and critical COVID-19 in hospitalized pregnancies: a US cohort study. Am J Obstet Gynecol MFM 2020. Doi: 10.1016/j.ajogmf.2020.100134

12 Yan J, Guo J, Fan C, et al. Coronavirus disease 2019 (COVID-19) in pregnant women: a report based on 116 cases. Am J Obstet Gynecol 2020. Doi: 10.1016/j.ajog.2020.04.014

13 Richardson S, Hirsch JS, Narasimhan M, et al; and the Northwell COVID-19 Research Consortium. Presenting characteristics, comorbidities, and outcomes among 5700 patients hospitalized with COVID-19 in the New York City area. JAMA 2020. Doi: $10.1001 /$ jama.2020.6775

14 Blitz MJ, Grünebaum A, Tekbali A, et al. Intensive care unit admissions for pregnant and non-pregnant women with COVID-19. Am J Obstet Gynecol 2020;S0002-9378(20)30528-7 . Doi: 10.1016/j.ajog.2020.05.004

$15 \mathrm{Wu}$ Z, McGoogan JM. Characteristics of and important lessons from the coronavirus disease 2019 (COVID-19) outbreak in China: summary of a report of 72314 cases from the Chinese center for disease control and prevention. JAMA 2020. Doi: 10.1001/jama.2020.2648

16 Chen H, Guo J, Wang C, et al. Clinical characteristics and intrauterine vertical transmission potential of COVID-19 infection in nine pregnant women: a retrospective review of medical records. Lancet 2020;395(10226):809-815

17 Goldenberg RL, Culhane JF, Iams JD, Romero R. Epidemiology and causes of preterm birth. Lancet 2008;371(9606):75-84

18 Zhou F, Yu T, Du R, et al. Clinical course and risk factors for mortality of adult inpatients with COVID-19 in Wuhan, China: a retrospective cohort study. Lancet 2020;395(10229):1054-1062

19 Saigal S, Doyle LW. An overview of mortality and sequelae of preterm birth from infancy to adulthood. Lancet 2008;371 (9608):261-269 